\title{
Curvature and isocurvature perturbations in a three-fluid model of curvaton decay
}

\author{
Sujata Gupta ${ }^{1}$, Karim A. Malik ${ }^{2,3}$ and David Wands ${ }^{1}$ \\ ${ }^{1}$ Institute of Cosmology and Gravitation, University of Portsmouth, Portsmouth PO1 2EG, United Kingdom \\ ${ }^{2}$ GRECO, Institut d'Astrophysique de Paris, C.N.R.S., 98bis Boulevard Arago, 75014 Paris, France \\ ${ }^{3}$ Physics Department, Lancaster University, Lancaster LA1 4YB, UK
}

(Dated: February 2, 2008)

\begin{abstract}
We study the evolution of the cosmological perturbations after inflation in curvaton models where the non-relativistic curvaton decays into both radiation and a cold dark matter component. We calculate the primordial curvature and correlated isocurvature perturbations inherited by the radiation and cold dark matter after the curvaton has decayed. We give the transfer coefficient in terms of the initial curvaton density relative to the curvaton decay rate.
\end{abstract}

PACS numbers: $98.80 . \mathrm{Cq}$

PU-ICG-03/43, astro-ph/0311562 v2

\section{INTRODUCTION}

Observational results obtained by a variety of recent cosmic microwave background and large scale structure experiments have greatly improved our picture of the Universe on the largest observable scales. These observations are consistent with a primordial spectrum of adiabatic, Gaussian, scale-independent perturbations [1, 2, 3, 4, 45, 6, , 7, 8, 9]. An attractive explanation is that these primordial perturbations in the early radiation-dominated era originate from quantum fluctuations in an earlier inflationary era where small scale vacuum fluctuations can be stretched up to arbitrarily large scales 10]. Although it is often assumed that primordial perturbations originate from adiabatic fluctuations of a slowly-rolling scalar field driving the inflationary expansion (the inflaton), any light scalar field (with effective mass less than the Hubble scale) will acquire an almost scale-invariant spectrum during an almost exponential expansion. Whether or not these isocurvature fluctuations in non-inflaton fields produce a primordial curvature perturbation depends upon whether or not the local value of that scalar field subsequently affects the local radiation density [11, 12].

In the curvaton scenario 13, 14, 15 a light, weakly coupled scalar field remains decoupled from the inflaton during inflation and remains decoupled after the end of inflation. Once the Hubble scale drops below the effective mass of the curvaton, the field oscillates and eventually decays. But if the decay is late enough then the density of the non-relativistic curvaton may have grown large enough to contribute significantly (more than about 1\%) to the total energy density of the universe. Then the local radiation density after curvaton decay depends upon the local value of the curvaton at the end of inflation and it is the initial isocurvature perturbation of the curvaton field that determines the primordial density perturbation. This scenario leads to new possibilities for building particle physics models of inflation [16] and qualitatively different constraints upon the inflationary parameters [17].

In the conventional slow-roll inflaton scenario there is a remarkable simplification when calculating observational constraints: one can calculate the primordial perturbations directly in terms of the inflaton field fluctuations during inflation, independently of the detailed physics of the end of inflation, reheating and the subsequent radiation-dominated era. This is because the inflaton fluctuations describe adiabatic perturbations about the background homogeneous cosmology on large scales and one can define a conserved curvature perturbation $\zeta$ directly from energy conservation [18, 19].

By contrast, fluctuations in the curvaton field describe non-adiabatic perturbations about the background cosmology and the total curvature perturbation $\zeta$ is no longer conserved on large scales. The non-adiabatic nature of the initial perturbations leaves open the possibility of residual isocurvature perturbations and detectable non-Gaussianity in the curvaton scenario [14, 15, 20, 21]. One can define individual curvature perturbations for the radiation and curvaton fluids that remain approximately conserved so long as the fluids can be assumed to be non-interacting [18, 19]. This has been used to derive an approximate calculation of the resulting primordial perturbations in the curvaton scenario in terms of the curvaton density at the decay time in what has been called the sudden-decay approximation [14, 20].

However an accurate calculation of the resulting primordial perturbations requires numerical solution of the perturbation equations. This was performed in [22] for a two-fluid model where non-relativistic curvaton particles decay into radiation. In this paper we extend this analysis to a three-fluid system to include the possibility that the curvaton also decays into non-relativistic cold dark matter $(\mathrm{CDM})$. This allows us to calculate the residual isocurvature perturbation that may be inherited by the matter in this scenario. See Ref. [15] for a numerical solution of the perturbed curvaton equations in a field theory description. Analytic estimates of the amplitude of the residual isocurvature perturbation in other models for the CDM were given in Ref. [21].

In section III we set up the coupled evolution equations for a system of dimensionless variables describing the 
evolution of the background homogeneous cosmology. We then give the coupled evolution equations for linear density perturbations in each fluid on uniform-curvature hypersurfaces in section III In an appendix we present equations describing the evolution of curvature and isocurvature perturbations in manifestly gauge-invariant form and discuss the appearance of singularities for some gauge-invariant quantities in a system such as this that includes energy transfer between fluids. This is seen to be due to a singular choice of hypersurface rather than a breakdown of perturbation theory.

The primordial perturbations that result from an initial curvaton perturbation can be characterised by a transfer coefficient that is a unique function of a single dimensionless parameter that specifies the initial density of the curvaton relative to the curvaton decay rate. We give an accurate analytic approximation (to better than 1\%) for the curvature transfer coefficient. The same coefficient also determines non-Gaussianity of the primordial perturbations [14] and, in our three-fluid model, the amplitude of the residual isocurvature perturbation. We are able to derive, as an exact result, a simple consistency relation between the ratio of isocurvature to curvature perturbations and the non-Gaussianity of the primordial perturbations, which was previously derived only using the sudden-decay approximation.

We present our conclusions in section IV

\section{BACKGROUND EQUATIONS AND DECAY}

In this section we will first review the governing equations of the background quantities in a Friedman-RobertsonWalker (FRW) universe and then find the evolution equations for the perturbations for three interacting fluids, following Ref. 22]. We begin the analysis after the end of inflation when the vacuum energy driving inflation has decayed into radiation. We assume that at that time the curvaton density is much smaller than the total density, consistent with the assumption that curvaton fluctuations are initially isocurvature perturbations.

The radiation $(\gamma)$ is a relativistic fluid with pressure $P_{\gamma}=\rho_{\gamma} / 3$. We model the other two components, the curvaton $(\sigma)$ and the cold dark matter $(m)$, as non-relativistic fluids, $P_{\sigma}=P_{m}=0$. This is a good description of the classical field dynamics once the Hubble rate has dropped below the effective mass of the curvaton so that the curvaton field begins coherent oscillations.

The evolution of a flat background FRW universe is governed by the Friedmann constraint and the continuity equation, respectively,

$$
\begin{aligned}
H^{2} & =\frac{8 \pi G}{3} \rho, \\
\dot{\rho} & =-3 H(\rho+P),
\end{aligned}
$$

where the dot denotes a derivative with respect to coordinate time $t, H$ is the Hubble parameter, and $\rho$ and $P$ are the total energy density and the total pressure, related to the density and pressure of the component fluids by

$$
\sum_{\alpha} \rho_{\alpha}=\rho, \quad \sum_{\alpha} P_{\alpha}=P .
$$

The continuity equation for each individual fluid in the background can be written as [23]

$$
\dot{\rho}_{\alpha}=-3 H\left(\rho_{\alpha}+P_{\alpha}\right)+Q_{\alpha}
$$

where $Q_{\alpha}$ describes the energy transfer per unit time to the $\alpha$-fluid.

We assume the curvaton can decay into both radiation and cold dark matter, described by the two decay rates, $\Gamma_{1}$ and $\Gamma_{2}$ respectively. For simplicity we assume the decay rates are fixed constants. This gives the background energy transfer as

$$
\begin{aligned}
Q_{\sigma} & =-\left(\Gamma_{1}+\Gamma_{2}\right) \rho_{\sigma}, \\
Q_{\gamma} & =\Gamma_{1} \rho_{\sigma}, \\
Q_{\mathrm{m}} & =\Gamma_{2} \rho_{\sigma} .
\end{aligned}
$$

The cold dark matter in this model is assumed to be a non-relativistic product of curvaton decay, which does not interact with radiation even at these early times. $\Gamma_{2} / \Gamma_{1}$ determines the energy density of cold dark matter particles relative to the radiation produced by curvaton decay. For the CDM to remain subdominant until matter-radiation equality at $T_{\mathrm{eq}} \simeq 1 \mathrm{eV}$ we require

$$
\frac{\Gamma_{2}}{\Gamma_{1}} \lesssim\left[\frac{\Omega_{\mathrm{m}}}{\Omega_{\gamma}}\right]_{\text {decay }}=\frac{T_{\mathrm{eq}}}{T_{\text {decay }}}<10^{-6}
$$


The final inequality follows from requiring that the curvaton decays before primordial nucleosynthesis $\left(T_{\text {decay }}>\right.$ $1 \mathrm{MeV})$, after which time any significant entropy production would spoil theoretical predictions of the primordial abundance of light elements.

The background energy transfer must always obey the constraint

$$
\sum_{\alpha} Q_{\alpha}=0
$$

It is useful to rewrite the background equations (4), in terms of dimensionless density parameters

$$
\Omega_{\sigma} \equiv \frac{\rho_{\sigma}}{\rho}, \quad \Omega_{\gamma} \equiv \frac{\rho_{\gamma}}{\rho}, \quad \Omega_{\mathrm{m}} \equiv \frac{\rho_{\mathrm{m}}}{\rho},
$$

which gives the evolution equations

$$
\begin{aligned}
& \Omega_{\sigma}^{\prime}=\Omega_{\sigma}\left(\Omega_{\gamma}-\frac{\Gamma_{1}+\Gamma_{2}}{H}\right), \\
& \Omega_{\gamma}^{\prime}=\Omega_{\sigma} \frac{\Gamma_{1}}{H}+\Omega_{\gamma}\left(\Omega_{\gamma}-1\right), \\
& \Omega_{\mathrm{m}}^{\prime}=\Omega_{\sigma} \frac{\Gamma_{2}}{H}+\Omega_{\mathrm{m}} \Omega_{\gamma},
\end{aligned}
$$

where a dash denotes the differentiation with respect to the number of e-foldings, $N \equiv \int H d t$.

The Friedmann constraint (11) then yields

$$
\Omega_{\sigma}+\Omega_{\gamma}+\Omega_{\mathrm{m}}=1
$$

The evolution of the inverse Hubble parameter is governed by

$$
\left(\frac{1}{H}\right)^{\prime}=\left(1+\frac{1}{3} \Omega_{\gamma}\right)\left(\frac{3}{2 H}\right) .
$$

Hence we have an autonomous dynamical system consisting of the four Eqs. (11) 13), and (15), but the constraint (14) makes one of them redundant.

\section{A. Numerical solutions}

In Fig 1 we show a phase-plane which plots the evolution of the curvaton energy density against the dimensionless decay rate, $0<\Gamma_{1} /\left(\Gamma_{1}+H\right)<1$. Note that for $\Gamma_{2} \ll \Gamma_{1}$ the evolution of $\Omega_{\sigma}$ is effectively independent of $\Gamma_{2}$ and the phase-plane shown is identical to that studied in Ref. [22].

All trajectories in the phase-plane in Fig 1 start close to the origin with $\Omega_{\sigma} \ll 1$ and $H \gg \Gamma_{1}$. From the limiting form of Eqs. (11) and (14) for $\Omega_{\gamma} \simeq 1$ we can see that $\Omega_{\sigma} \propto H^{-1 / 2} \propto e^{N}$ [22]. Thus different trajectories can be identified by different values of the dimensionless parameter

$$
p \equiv\left[\Omega_{\sigma}\left(\frac{H}{\Gamma_{1}}\right)^{\frac{1}{2}}\right]_{\text {initial }} .
$$

If we consider the initial conditions for the fluid model to be set when the curvaton begins to oscillate $\left(H_{\text {in }}=m_{\sigma}\right)$ then we can write

$$
p=\frac{8 \pi\left\langle\sigma^{2}\right\rangle_{\text {in }}}{3 M_{\mathrm{Pl}}^{2}}\left(\frac{m_{\sigma}}{\Gamma_{1}}\right)^{1 / 2}
$$

The curvaton vacuum expectation value is bounded, $\left\langle\sigma^{2}\right\rangle_{\text {in }}<M_{\mathrm{Pl}}^{2}$, as we work in the regime where there is no phase of curvaton-driven inflation [17]. Nonetheless $p$ can be large if the curvaton decay rate is slow, e.g., $m_{\sigma} / \Gamma_{1} \sim\left(M_{\mathrm{Pl}} / m_{\sigma}\right)^{2}$ for gravitational strength decay.

We show in Figures 2 to 4 examples of the time-evolution of the three fluid energy densities along different trajectories. Figure 2 a) corresponds to trajectory A of the phase-plane Figure 11 The system begins radiation dominated but as time passes the curvaton density, with a dust equation of state, grows relative to the radiation density. The 


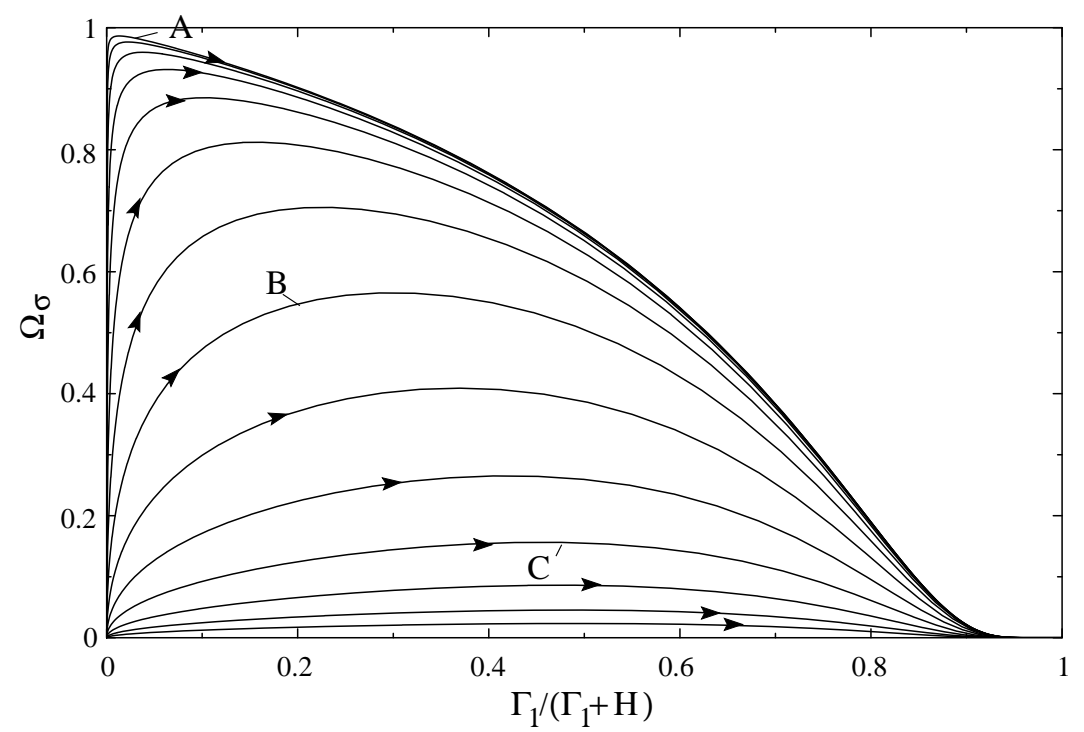

FIG. 1: The phase plot shows the evolution of the dimensionless energy density, $\Omega_{\sigma}$, for different values of the dimensionless parameter, p, defined in Eq. (16), varying from $3.15 \times 10^{3}$ (top line) to $3.15 \times 10^{-3}$ (bottom line). For all of the lines $\Gamma_{2} \ll \Gamma_{1}$. The evolution of the densities of all three fluids are plotted below in Figs. 2 to 4 for the trajectories, marked A, B and C.

energy density of the curvaton then rapidly decays when $H \sim \Gamma_{1}$. In this example there is a period of curvaton domination $\left(\Omega_{\sigma} \simeq 1\right)$ before the curvaton decays.

Figure B(a), corresponding to trajectory B of the phase-plane figure (11), has a lower value the parameter $p$, so the curvaton density does not climb as high before it decays away. Figure [4(a), corresponding to trajectory $\mathrm{C}$ of the phase-plane figure (11), has the lowest value of $p$ of the three plots and the ratio of initial curvaton density to the decay rate is low enough that there is no period of curvaton domination in this example.

\section{PERTURBED EQUATIONS}

The evolution equation for density perturbations in the $\alpha$ fluid is given on large scales and on uniform-curvature hypersurfaces by $[22]$

$$
\dot{\delta \rho_{\alpha}}+3 H\left(\delta \rho_{\alpha}+\delta P_{\alpha}\right)+Q_{\alpha} \frac{\delta \rho}{2 \rho}-\delta Q_{\alpha}=0
$$

where $\delta \rho_{\alpha}, \delta P_{\alpha}$ and $\delta Q_{\alpha}$ are the density perturbations, the pressure perturbation, and the energy transfer perturbation of the $\alpha$-fluid. $\delta \rho$ is the total density perturbation on the uniform-curvature hypersurfaces and appears in the equation for the perturbed energy transfer due to the gravitational time dilation [22].

The perturbed energy transfer (assuming the physical decay rates are unperturbed) is given by,

$$
\begin{aligned}
\delta Q_{\sigma} & =-\left(\Gamma_{1}+\Gamma_{2}\right) \delta \rho_{\sigma}, \\
\delta Q_{\gamma} & =\Gamma_{1} \delta \rho_{\sigma}, \\
\delta Q_{\mathrm{m}} & =\Gamma_{2} \delta \rho_{\sigma} .
\end{aligned}
$$

We will evolve the perturbed conservation equations (18), which can be written in terms of the dimensionless density 


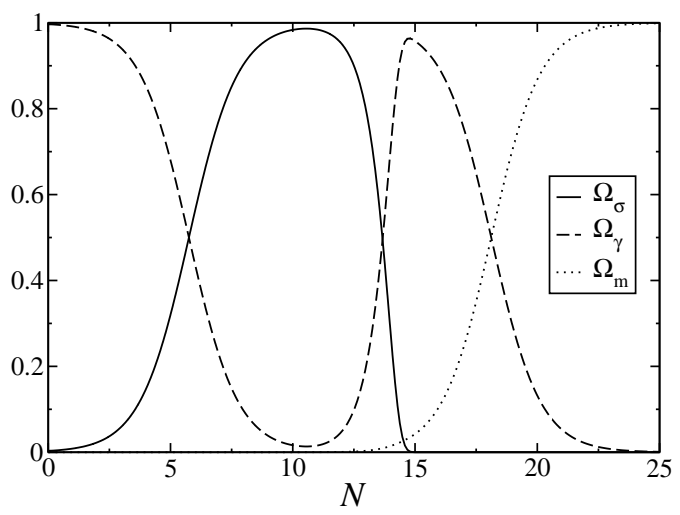

(a)

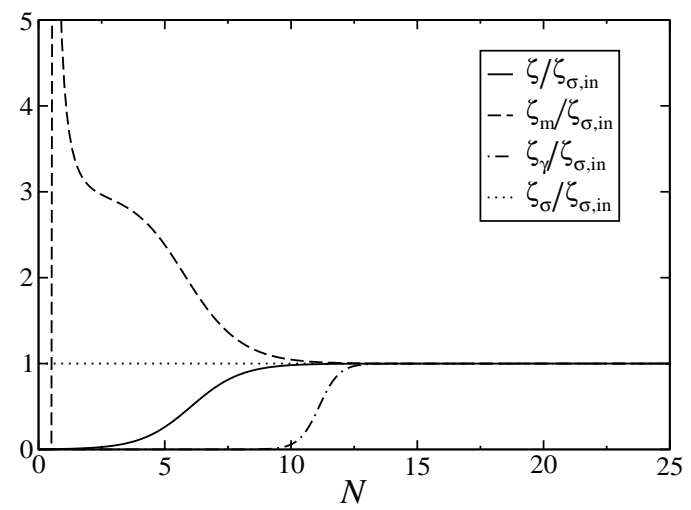

(b)

FIG. 2: (a) The evolution of $\Omega_{\sigma}$ (solid), $\Omega_{\gamma}$ (dashed) and $\Omega_{\mathrm{m}}$ (dotted) against e-foldings $N$ of line A of Fig 1 The initial value of $\Omega_{\sigma}$ is $10^{-2.5}$, and $\Gamma_{1}=10^{-10}$ and $\Gamma_{2}=10^{-12}$. (b) The evolution of $\zeta_{\sigma} / \zeta_{\sigma, \text { in }}\left(\right.$ dotted), $\zeta_{\gamma} / \zeta_{\sigma, \text { in }}\left(\right.$ dot-dashed), $\zeta_{\mathrm{m}} / \zeta_{\sigma, \text { in }}$ (dashed) and the total curvature perturbation, $\zeta / \zeta_{\sigma, \text { in }}$ (solid), against e-foldings $N$ of line A of Fig 1

parameters as

$$
\begin{aligned}
& \delta \rho_{\sigma}^{\prime}+\left(3+\frac{\Gamma_{1}+\Gamma_{2}}{H}\right) \delta \rho_{\sigma}-\frac{\Gamma_{1}+\Gamma_{2}}{H} \Omega_{\sigma} \frac{\delta \rho}{2}=0, \\
& \delta \rho_{\gamma}^{\prime}+4 \delta \rho_{\gamma}-\frac{\Gamma_{1}}{H}\left(\delta \rho_{\sigma}-\Omega_{\sigma} \frac{\delta \rho}{2}\right)=0 \\
& \delta \rho_{\mathrm{m}}^{\prime}+3 \delta \rho_{\mathrm{m}}-\frac{\Gamma_{2}}{H}\left(\delta \rho_{\sigma}-\Omega_{\sigma} \frac{\delta \rho}{2}\right)=0 .
\end{aligned}
$$

where a prime denotes derivatives with respect to $N$.

Written in this form it is evident that the linear evolution of the density perturbations depends only upon the dimensionless background variables $\Omega_{\alpha}$ and decay rates $\Gamma_{\alpha} / H$. Hence the final amplitude of the density perturbations,

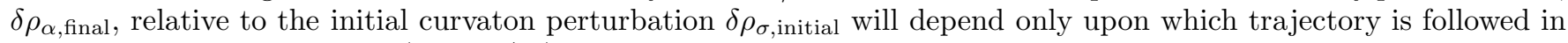
the background phase-space $\left(\Omega_{\alpha}, \Gamma_{\alpha} / H\right)$. They will not depend on dimensional quantities such as $H_{\text {initial }}$. In fact we will show that the large-scale perturbations after the curvaton has decayed can be written simply in terms of the initial curvaton perturbation and a function of the dimensionless parameter $p$ defined in Eq. (16). 


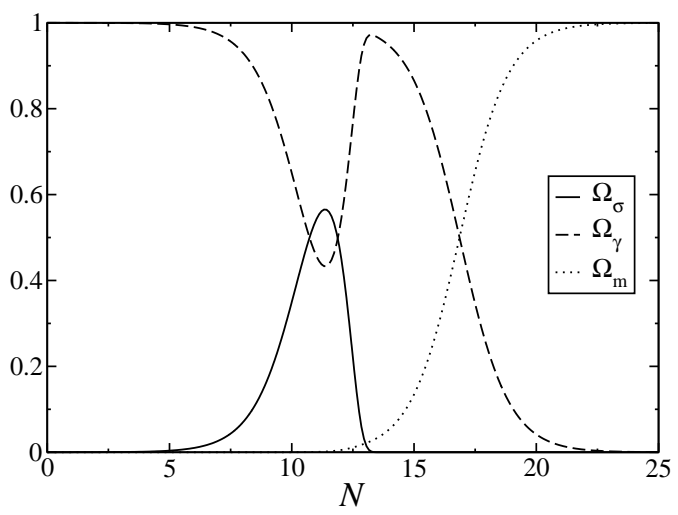

(a)

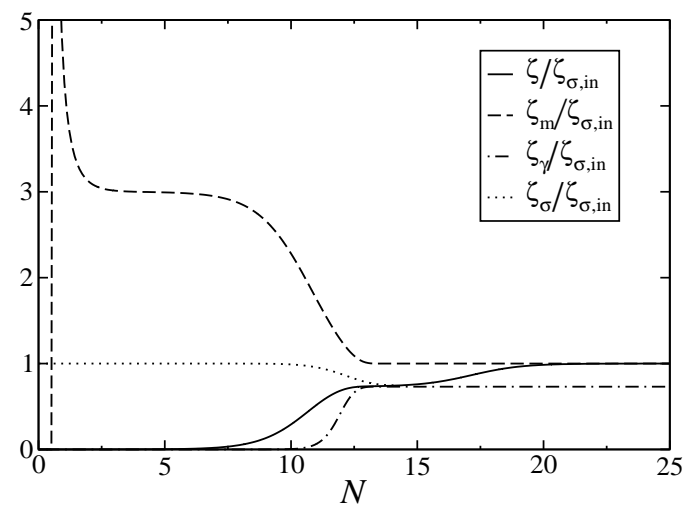

(b)

FIG. 3: (a) The evolution of $\Omega_{\sigma}$ (solid), $\Omega_{\gamma}$ (dashed) and $\Omega_{\mathrm{m}}$ (dotted) against e-foldings $N$ of line B of Fig 1 The initial value of $\Omega_{\sigma}$ is $10^{-4.6}$. $\Gamma_{1}$ and $\Gamma_{2}$ are the same as in Fig[2 (b) The evolution of $\zeta_{\sigma} / \zeta_{\sigma, \text { in }}$ (dotted), $\zeta_{\gamma} / \zeta_{\sigma, \text { in }}\left(\right.$ dot-dashed), $\zeta_{\mathrm{m}} / \zeta_{\sigma, \text { in }}$ (dashed) and the total curvature perturbation, $\zeta / \zeta_{\sigma, \text { in }}$ (solid), against e-foldings $N$ of line B of Fig 1

\section{A. Curvature and isocurvature perturbations}

The gauge-invariant variable, $\zeta$, introduced by Bardeen [24, 25], is commonly used to describe the large-scale curvature perturbation. It is equivalent to the curvature perturbation of uniform-density hypersurfaces and remains constant on super-horizon scales for adiabatic perturbations. This quantity is simply related to the total density perturbation on uniform-curvature hypersurfaces [18, 25]

$$
\zeta=-H \frac{\delta \rho}{\dot{\rho}} .
$$

We can also define the curvature perturbation on uniform $\alpha$-fluid density hypersurfaces, $\zeta_{\alpha}$, which is related to the $\alpha$-fluid density on uniform-curvature hypersurfaces 18

$$
\zeta_{\alpha}=-H \frac{\delta \rho_{\alpha}}{\dot{\rho}_{\alpha}} .
$$

The total density perturbation is just the sum of the density perturbations of the individual fluids, $\delta \rho=\sum_{\alpha} \delta \rho_{\alpha}$, and hence the total curvature perturbation $\zeta$ is the weighted sum of the individual perturbations [18],

$$
\zeta=\sum_{\alpha} \frac{\dot{\rho}_{\alpha}}{\dot{\rho}} \zeta_{\alpha} .
$$




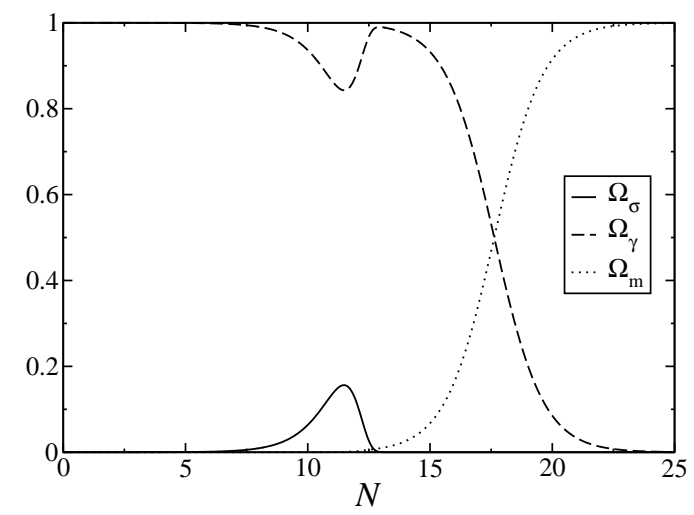

(a)

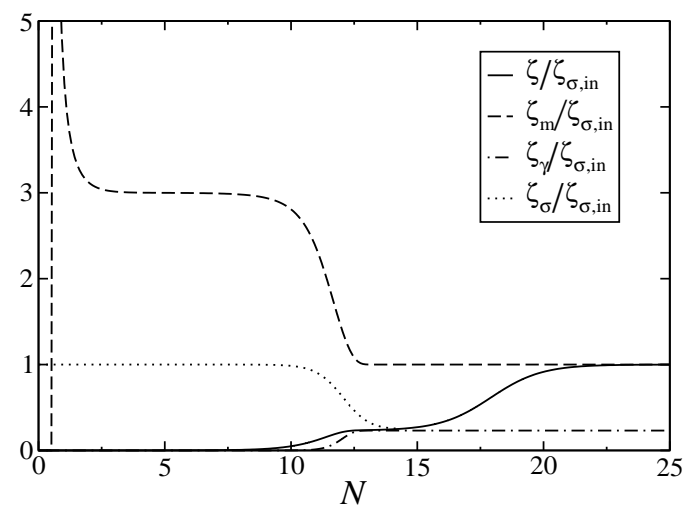

(b)

FIG. 4: (a) The evolution of $\Omega_{\sigma}$ (solid), $\Omega_{\gamma}$ (dashed) and $\Omega_{\mathrm{m}}$ (dotted) against e-foldings $N$ of line C of Fig 1 The initial value of $\Omega_{\sigma}$ is $10^{-5.5}$. $\Gamma_{1}$ and $\Gamma_{2}$ are the same as in Fig[2 (b) The evolution of $\zeta_{\sigma} / \zeta_{\sigma, \text { in }}$ (dotted), $\zeta_{\gamma} / \zeta_{\sigma, \text { in }}\left(\right.$ dot-dashed), $\zeta_{\mathrm{m}} / \zeta_{\sigma, \text { in }}$ (dashed) and the total curvature perturbation, $\zeta / \zeta_{\sigma}$,in (solid), against e-foldings $N$ of line $\mathrm{C}$ of Fig 1

The difference between any two individual curvature perturbations, $\zeta_{\alpha}$ and $\zeta_{\beta}$, describes an isocurvature or entropy perturbation [18, 22]

$$
\mathcal{S}_{\alpha \beta}=3\left(\zeta_{\alpha}-\zeta_{\beta}\right)
$$

Note that the definition of $\zeta_{\alpha}$ becomes singular whenever $\dot{\rho}_{\alpha}=0$. For instance the physical density of cold dark matter is initially vanishing, but growing due to the decay of the curvaton $\left(\dot{\rho}_{m}>0\right)$. However after the curvaton has completely decayed, the matter density is diluted by the expansion $\left(\dot{\rho}_{m}<0\right)$. At one instant we have $\dot{\rho}_{m}=0$ and $\zeta_{m}$ is ill-defined. This has been previously noted in the case of an oscillating scalar field at stationary values of the field [26]. It is a consequence of the uniform $\alpha$-density hypersurfaces becoming ill-defined, and does not signal the breakdown of perturbation theory, as long as we can find some gauge where the hypersurfaces are well-defined and perturbations remain small (see Appendix).

In this case we will numerically solve for the fluid density perturbations on uniform-curvature hypersurfaces. Fig. 5 shows an example of the evolution of the density perturbations in equations (22) to (24) above, showing that they do indeed remain well-defined throughout the evolution.

From the density perturbations we then construct the curvature perturbations, Eq.(25) and (26), which gives

$$
\zeta_{\sigma}=\frac{8 \pi G}{3 H} \frac{\delta \rho_{\sigma}}{\Omega_{\sigma}\left(3 H+\Gamma_{1}+\Gamma_{2}\right)},
$$




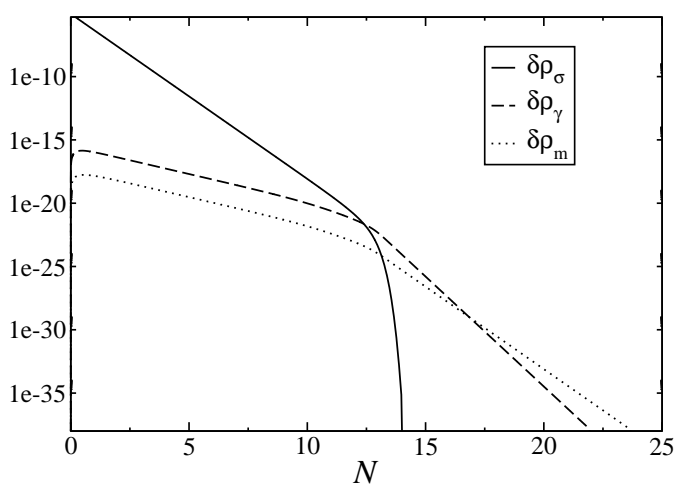

FIG. 5: The evolution of the density perturbations on uniform-curvature hypersurfaces, $\delta \rho_{\sigma}$ (solid), $\delta \rho_{\gamma}$ (dashed) and $\delta \rho_{\mathrm{m}}$ (dotted), against e-foldings $N$ along the background trajectory B in Fig 1

$$
\begin{gathered}
\zeta_{\gamma}=-\frac{8 \pi G}{3 H} \frac{\delta \rho_{\gamma}}{\left(-4 H \Omega_{\gamma}+\Gamma_{1} \Omega_{\sigma}\right)}, \\
\zeta_{\mathrm{m}}=-\frac{8 \pi G}{3 H} \frac{\delta \rho_{\mathrm{m}}}{\left(-3 H \Omega_{\mathrm{m}}+\Gamma_{2} \Omega_{\sigma}\right)},
\end{gathered}
$$

At late times, after the curvaton has completely decayed the fluids are non-interacting and have monotonically decreasing energy densities (diluted by the expansion) so the curvature perturbations, $\zeta_{\alpha}$, are well-defined, and constant on large scales. Observations of, for example, the CMB directly constrain the amplitude of the total curvature and isocurvature perturbations. On large angular scales the temperature perturbations on the surface of last-scattering are given by the Sachs-Wolfe effect [6, 7, 27]

$$
\left(\frac{\delta T}{T}\right)_{\mathrm{lss}}=\frac{1}{5}\left(-\zeta_{\gamma}-2 \mathcal{S}_{m \gamma}\right)_{\mathrm{lss}}
$$

\section{B. Numerical results}

We have numerically evolved the coupled system of background equations (11)-(13) and (15) and first-order perturbations (22)-(24). In each case we begin with a smooth $\left(\zeta_{\gamma, \text { in }}=0\right)$, dominant radiation fluid $\left(\Omega_{\gamma, \text { in }} \simeq 1\right)$ and a subdominant curvaton fluid $\left(\Omega_{\sigma, \text { in }} \ll 1\right)$ with an initial perturbation $\zeta_{\sigma, \text { in }} \neq 0$. The cold dark matter density is initially zero $\left(\Omega_{\mathrm{m}, \text { in }}=0, \zeta_{\mathrm{m} \text {,in }}=0\right)$, and is produced solely from curvaton decay. The initial density perturbations on uniform-curvature hypersurfaces are given in terms of $\zeta_{\alpha, \text { in }}$ from Eqs. (29)-(31).

In all cases we start the evolution at some high energy scale, with a large value of the Hubble parameter, characterised by a small initial value of the Hubble time relative to the decay time, $\Gamma_{1} / H_{\text {in }} \ll 1$. The subsequent evolution is not directly affected by the precise value of $\Gamma_{1} / H_{\text {in }}$, but rather by the parameter $p$ defined in Eq. (16) which characterises the phase-space trajectory. In particular the final values of the curvature and isocurvature perturbations are a function solely of the parameter $p$.

Figures 2(b), 3(b) and 4(b) show the evolution of the large-scale curvature perturbations for the three fluids along three different background trajectories.

\section{Radiation}

The radiation curvature perturbation $\zeta_{\gamma}$ grows as the curvaton decays, and reaches an equilibrium point in the phase-space where $\zeta_{\gamma}=\zeta_{\sigma}$ [22]. The final value for $\zeta_{\gamma}$ relative to the initial value of $\zeta_{\sigma \text {,in }}$ depends upon the relative density of the curvaton at the decay epoch, $\Omega_{\sigma \text {,decay }}$. This determines the proportion of the final radiation that comes from the decay of the perturbed curvaton. 


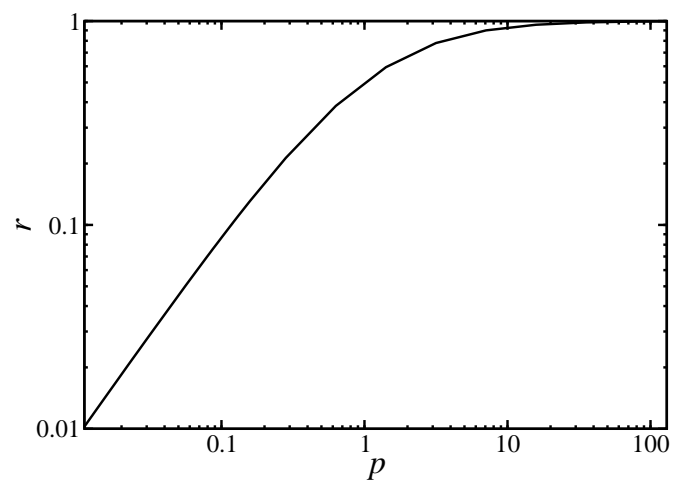

FIG. 6: Curvature transfer coefficient $r \equiv \zeta_{\gamma, \text { out }} / \zeta_{\sigma, \text { in }}$, plotted against background parameter $p \equiv\left[\Omega_{\sigma}\left(H / \Gamma_{1}\right)^{1 / 2}\right]_{\text {in }}$.

For example, in Fig. 2(a), corresponding to trajectory A of Fig. 1 the curvaton comes to dominate $\left(\Omega_{\sigma} \simeq 1\right)$ before the decay epoch. Thus almost all the radiation at late times comes from the decay of the curvaton and hence, in Fig. 2(b), the radiation fluid at late times inherits the initial curvaton perturbation, $\zeta_{\gamma} \simeq \zeta_{\sigma \text {,in }}$. Compare this with Fig. [1 corresponding to trajectory $\mathrm{C}$ of the Fig. 10 In this case the curvaton decays while $\Omega_{\sigma} \ll 1$ and the proportion of the final radiation energy density which results from the decay of the perturbed curvaton component is much smaller in this picture.

We define $r$ to be the ratio between the curvature perturbation of the radiation component at the end of the calculation (when all of the curvaton component has decayed away) to the initial curvaton perturbation,

$$
\zeta_{\gamma, \text { out }}=r \zeta_{\sigma, \text { in }} .
$$

We find that $r$ is solely a function of the choice of trajectory in the background phase-space, Fig. (1). Each trajectory is uniquely identified in terms of the initial data by the value of the parameter $p$ defined in Eq. (16).

We plot $r$ against $p$ in Fig. (6). At small values of $p$ this approaches a linear function

$$
r \simeq 0.924 p
$$

see Fig. (7). This coincides with the sudden-decay approximation calculated in Ref. [22] for $\Omega_{\sigma, \text { in }} \ll 1$. The linear approximation is good to better than $1 \%$ up to $p=0.011(r=0.0108)$ and better than $10 \%$ up to $p=0.11(r=0.10)$.

At larger values of $p$ the transfer coefficient $r(p)$ turns over and $r \rightarrow 1$ for $p \gg 1$. The full function is well fit by

$$
r_{\text {fit }}(p)=1-\left(1+\frac{0.924}{q} p\right)^{-q}
$$

where, by fitting over the range $10^{-2}<p<10^{2}$, we find $q=1.24$. This gives a fit to the numerical calculation of $r(p)$ to better than $0.2 \%$ for any value of $p$.

\section{Cold Dark Matter}

In our model the universe starts with an initial cold dark matter density, $\rho_{m}$, equal to zero which then grows due to curvaton decay, but eventually starts decreasing due to cosmic expansion. As remarked earlier the curvature perturbation associated with the cold dark matter, $\zeta_{m}$, becomes ill-defined when $\dot{\rho}_{m}=0$. This occurs when $Q_{m}=$ $\Gamma_{2} \rho_{\sigma}=3 H \rho_{m}$, in Eq. (4) for $\alpha=m$. The divergence of $\zeta_{m}$ is clearly seen at early times (before the curvaton decays) in Figures 2(b), 3(b) and 4(b).

The curvature perturbation $\zeta_{m}$ is initially time-dependent due to the energy transfer from the perturbed curvaton, but settles down to a constant value at late times, once the curvaton has completely decayed $\Gamma_{1}+\Gamma_{2} \gg H$. We find that if the cold dark matter is produced solely from curvaton decay, it inherits a curvature perturbation $\zeta_{m, \text { out }}$ at late times equal to the initial curvaton perturbation, $\zeta_{\sigma, \text { in }}$. 


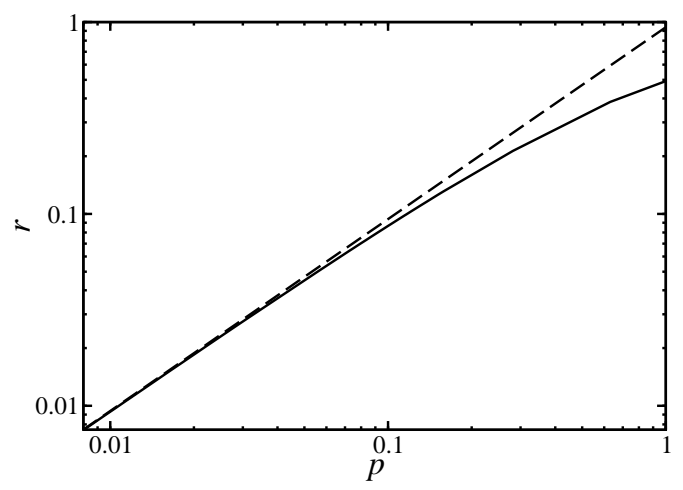

FIG. 7: Curvature transfer coefficient $r$, defined in Eq. (33), plotted against parameter $p$, defined in Eq. (16), with a linear fit $r=0.924 p$ for $p \ll 1$. This linear fit is accurate to within $1 \%$ for $p<0.011$.

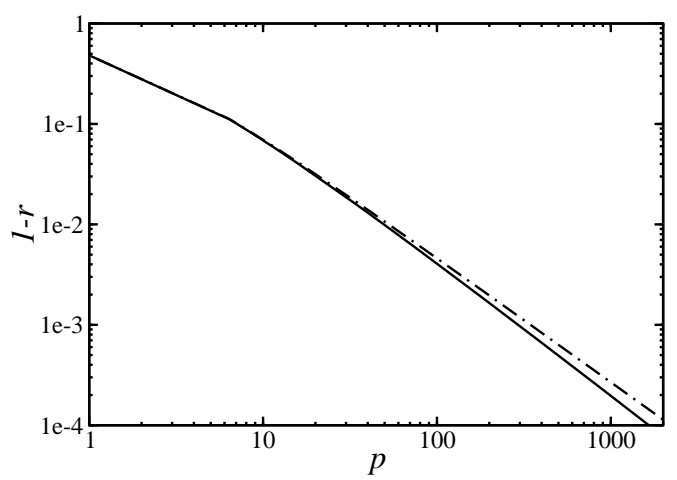

FIG. 8: Isocurvature transfer coefficient, 1-r, defined in Eq. [33), plotted against parameter $p$, defined in Eq. (16). Also shown (dot-dashed line) is the fitting function $1-r_{\text {fit }}$, defined in Eq. (35). This is accurate to within $1 \%$ for $p<8.14$ (corresponding to $1-r>0.086)$.

This simple result can be derived by considering a composite energy density constructed from the CDM density and a fixed fraction of the curvaton density:

$$
\rho_{\text {comp }} \equiv \rho_{\mathrm{m}}+\frac{\Gamma_{2}}{\Gamma_{1}+\Gamma_{2}} \rho_{\sigma}
$$

From Eqs. (5) and (7) we see that the corresponding energy transfer is zero, i.e.,

$$
Q_{\text {comp }} \equiv Q_{\mathrm{m}}+\frac{\Gamma_{2}}{\Gamma_{1}+\Gamma_{2}} Q_{\sigma}=0
$$

Thus we obtain from Eqs. (44) and (18) the standard evolution equations for the composite energy density and its perturbation:

$$
\begin{aligned}
\dot{\rho}_{\text {comp }} & =-3 H \rho_{\text {comp }} \\
\dot{\delta \rho_{\text {comp }}} & =-3 H \delta \rho_{\text {comp }} .
\end{aligned}
$$

Note that as both the curvaton and CDM are pressureless then so is the composite energy density, $P_{\text {comp }}=0$. Since we have a conserved energy density with a unique equation of state the corresponding curvature perturbation, defined by Eq. (26),

$$
\zeta_{\text {comp }}=\frac{\delta \rho_{\text {comp }}}{3 \rho_{\text {comp }}},
$$


is conserved on large scales 18,19 .

Initially $\rho_{\mathrm{m}}=0$ and $\delta \rho_{\mathrm{m}}=0$ and hence $\zeta_{\text {comp }}=\zeta_{\sigma, \text { in }}$. At late times $\rho_{\mathrm{m}} \gg\left(\Gamma_{2} /\left(\Gamma_{1}+\Gamma_{2}\right)\right) \rho_{\sigma}$, and $\zeta_{\text {comp }}=\zeta_{\mathrm{m} \text {,out }}$. Therefore

$$
\zeta_{\mathrm{m}, \mathrm{out}}=\zeta_{\sigma, \mathrm{in}}
$$

as we see in Figs. 2 , 3 and 4

\section{Curvature and isocurvature perturbations}

After the curvaton has decayed, $\left(\Omega_{\sigma} \rightarrow 0\right)$, we are left with one overall curvature perturbation, Eq. (27),

$$
\zeta=\frac{4 \Omega_{\gamma}}{4 \Omega_{\gamma}+3 \Omega_{\mathrm{m}}} \zeta_{\gamma}+\frac{3 \Omega_{\mathrm{m}}}{4 \Omega_{\gamma}+3 \Omega_{\mathrm{m}}} \zeta_{\mathrm{m}},
$$

and one relative isocurvature (entropy) perturbation between the radiation and cold dark matter, Eq. (28),

$$
\mathcal{S}_{\mathrm{m} \gamma}=3\left(\zeta_{\mathrm{m}}-\zeta_{\gamma}\right) .
$$

The primordial curvature perturbation is usually taken to be the curvature perturbation at, for instance, the epoch of primordial nucleosynthesis when the Universe is known to be radiation dominated $\left(\Omega_{\gamma} \gg \Omega_{\mathrm{m}}, \Omega_{\sigma}=0\right)$, and hence $\zeta \simeq \zeta_{\gamma}$.

We have seen that the primordial curvature perturbation relative to the initial curvaton perturbation is a function of the trajectory, $r(p)$ in Eq. (33),

$$
\left.\zeta\right|_{\text {nuc }} \equiv \zeta_{\gamma, \text { out }}=r \zeta_{\sigma, \text { in }},
$$

whereas the final cold dark matter curvature perturbation is identical to the initial curvaton perturbation, Eq. (41). Hence

$$
\left.\mathcal{S}\right|_{\text {nuc }} \equiv \mathcal{S}_{\mathrm{m} \gamma, \text { out }}=3(1-r) \zeta_{\sigma, \text { in }} .
$$

If $r=1$ then we obtain an adiabatic curvature perturbation after the curvaton has decayed and there is no late-time isocurvature perturbation.

Note that the fitting function $r_{\text {fit }}(p)$ given in Eq. (35) gives a good approximation, for $1-r$ (better than $1 \%$ for $r<0.914(p<8.14)$ or better than $10 \%$ for $r<0.993(p<66))$.

Therefore the primordial isocurvature and curvature perturbations are $100 \%$ correlated with their ratio fixed by $r(p)$ :

$$
\left.\frac{\mathcal{S}}{\zeta}\right|_{\text {nuc }}=\frac{3(1-r)}{r} .
$$

This expression was derived in Ref. [20] using the sudden-decay approximation. We have shown that it is in fact an exact expression when the CDM is produced directly from the decay of the curvaton, and $r$ is defined by Eq. (33).

\section{CONCLUSIONS}

In this paper we have studied the coupled evolution of background densities and linear perturbations of three interacting fluid components: radiation, a curvaton field and cold dark matter. We solve numerically the evolution equations for density perturbations on spatially flat hypersurfaces to calculate the spectrum of curvature and isocurvature (or relative entropy) perturbations that result from initial perturbations in the curvaton field.

Whereas the resulting radiation curvature perturbation, $\zeta_{\gamma, \text { out }}$, is dependent on the initial values of background parameters (such as curvaton density), we find the simple result that the final CDM curvature perturbation, $\zeta_{\mathrm{m} \text {,out }}$, is always exactly equal to the initial curvaton perturbation $\zeta_{\sigma \text {,in }}$, in a model where the CDM is directly produced by curvaton decay. We are able to demonstrate this analytically by constructing a conserved quantity, $\zeta_{\text {comp }}$, that at early times is equal to the curvaton perturbation and at late times is equal to the CDM curvature perturbation. In effect this solves the evolution of one degree of freedom and allows us to solve numerically only for $\zeta_{\gamma}$ as a function of input parameters. 
The background evolution of the fluids is described by a trajectories in phase-space distinguished by a dimensionless parameter $p=\left[\Omega\left(H / \Gamma_{1}\right)^{1 / 2}\right]_{\text {in }}$. The ratio $r$ between $\zeta_{\gamma, \text { out }}$ and $\zeta_{\sigma \text {,in }}$ is a function solely of this trajectory parameter $p$ and is well-fit by a simple numerical function given in Eq. (35). This coincides with the "sudden-decay approximation" [14, 22] for $p \ll 1$.

The resulting radiation and $\mathrm{CDM}$ perturbations after curvaton decay can then be used to predict the spectrum of $\mathrm{CMB}$ anisotropies in a given model where the CDM is produced directly as a curvaton decay product. CMB constraints are usually quoted in terms of the primordial curvature $\zeta_{\text {nuc }}$ and an isocurvature perturbation $\mathcal{S}_{\text {nuc }}$ around the epoch of primordial nucleosynthesis, leading to

$$
\left(\begin{array}{l}
\zeta \\
\mathcal{S}
\end{array}\right)_{\mathrm{nuc}}=\left(\begin{array}{cc}
1 & r / 3 \\
0 & (1-r)
\end{array}\right)\left(\begin{array}{l}
\zeta \\
\mathcal{S}
\end{array}\right)_{\mathrm{in}} .
$$

In the curvaton scenario $\zeta_{\text {in }}=\zeta_{\gamma, \text { in }}=0$ and $\mathcal{S}_{\text {in }}=3\left(\zeta_{\sigma, \text { in }}-\zeta_{\gamma, \text { in }}\right)=3 \zeta_{\sigma, \text { in }}$. Although the model we have studied describes the production of CDM directly from the curvaton decay, one would expect a similar result to hold for models where the net baryon or lepton number is directly produced from curvaton decay.

The matrix equation (47) is a particular case of the general form of the transfer matrix for curvature and isocurvature perturbations presented in Ref. [6, 28] where the contribution of the initial isocurvature perturbation to the resulting primordial curvature and isocurvature perturbation is model dependent. For instance in a curvaton scenario where the CDM is a thermal relic which decouples from radiation sometime after the curvaton has decayed we have instead

$$
\left(\begin{array}{l}
\zeta \\
\mathcal{S}
\end{array}\right)_{\text {nuc }}=\left(\begin{array}{cc}
1 & r / 3 \\
0 & 0
\end{array}\right)\left(\begin{array}{l}
\zeta \\
\mathcal{S}
\end{array}\right)_{\text {in }} .
$$

The matrix equations (47) and (48) using the numerically determined function $r(p)$ [or its approximate form $r_{\text {fit }}(p)$ ] enable one to relate $\mathrm{CMB}$ observations to curvaton model parameters such as the curvaton decay rate, $\Gamma$, and the curvaton density, $\rho_{\sigma}$, at some early time, characterised by the value of the Hubble rate $H$, and the initial spectrum of curvaton perturbations $\zeta_{\sigma}=(1 / 3) \delta \rho_{\sigma} / \rho_{\sigma}$.

For example, in the model where the CDM is directly produced by curvaton decay we confirm [14] that the relative amplitude of primordial isocurvature to curvature perturbations is a fixed ratio $3(1-r) / r$ which is a function solely of the background parameter $p$ defined in Eq. (16). This holds independently of the "sudden-decay approximation" previously used.

In any curvaton model the transfer coefficient $r$ is independent of scale in the large-scale limit (by definition) so that the scale-dependence of the primordial perturbation spectra are inherited directly from the scale dependence of the curvaton perturbation at the end of inflation [14, 28]

$$
\Delta n_{\sigma} \equiv \frac{d \ln \mathcal{P}_{\delta \sigma}}{d \ln k}=-2 \epsilon+2 \eta_{\sigma} .
$$

In this expression $\epsilon \equiv-\dot{H} / H^{2}$ is the usual dimensionless slow-roll parameter during inflation and $\eta_{\sigma} \equiv m_{\sigma}^{2} / 3 H^{2}$ is the effective mass of the curvaton relative to the Hubble scale during inflation. Both the curvature and isocurvature perturbation (if any) originate from initial curvaton perturbation and are thus completely correlated, independent of the physics of the curvaton decay. In the absence of any additional source for the isocurvature perturbations, the curvature and any isocurvature perturbations must share the same spectral tilt, given by Eq. (49).

Note that the curvature transfer coefficient $r$ can also be related to the non-Gaussianity of the primordial perturbations. If $r$ is small then the initial curvaton perturbation $\zeta_{\sigma \text {,in }}$ must be correspondingly large in order to produce a given amplitude of primordial curvature perturbation. This leads to a larger contribution from second-order perturbations in the curvaton density, which shows up in the non-linearity parameter [9, 29]

$$
f_{\mathrm{NL}} \approx \frac{5}{4 r},
$$

for $r \ll 1$ [14]. (See [30] for corrections when $r \sim 1$.) There is thus a possible consistency test of this curvaton model using two observables. Existing constraints on the relative amplitude of primordial isocurvature to curvature perturbations [7] constrain $r>0.9$ in models where the CDM is directly produced by curvaton decay, and hence limit the allowed non-Gaussianity. However in curvaton models which produce purely adiabatic perturbations $\left(\mathcal{S}_{\text {nuc }}=0\right)$, determinations of the non-linear parameter $f_{\mathrm{NL}}$ may be the only way to determine independently both the transfer parameter $r$ (and hence the model parameter $p$ ) and the initial amplitude of the curvaton perturbation $\zeta_{\sigma}$. 


\section{Acknowledgments}

The authors are grateful to David Lyth and Lorenzo Sorbo for useful comments. This work was supported by PPARC grants $P P A / G / S / 2000 / 00115, P P A / G / S / 2002 / 00098$, and $P P A / V / S / 2001 / 00544$. KAM was supported by a Marie Curie Fellowship under contract number HPMF-CT-2000-00981. DW is supported by the Royal Society.

[1] R. Trotta, A. Riazuelo and R. Durrer, Phys. Rev. Lett. 87, 231301 (2001) arXiv:astro-ph/0104017.

[2] M. Tegmark et al. [SDSS Collaboration], arXiv:astro-ph/0310723

[3] C. Gordon and K. A. Malik, arXiv:astro-ph/0311102

[4] P. Crotty, J. Garcia-Bellido, J. Lesgourgues and A. Riazuelo, Phys. Rev. Lett. 91, 171301 (2003) arXiv:astro-ph/0306286.

[5] H. V. Peiris et al., Astrophys. J. Suppl. 148, 213 (2003) arXiv:astro-ph/0302225.

[6] L. Amendola, C. Gordon, D. Wands and M. Sasaki, Phys. Rev. Lett. 88, 211302 (2002) arXiv:astro-ph/0107089.

[7] C. Gordon and A. Lewis, Phys. Rev. D 67, 123513 (2003) arXiv:astro-ph/0212248.

[8] J. Valiviita and V. Muhonen, Phys. Rev. Lett. 91, 131302 (2003) arXiv:astro-ph/0304175.

[9] E. Komatsu et al., Astrophys. J. Suppl. 148, 119 (2003) arXiv:astro-ph/0302223.

[10] A. R. Liddle and D. H. Lyth, "Cosmological Inflation And Large-Scale Structure", CUP (2000).

[11] S. Mollerach, Phys. Rev. D 42 (1990) 313.

[12] A. D. Linde and V. Mukhanov, Phys. Rev. D 56, 535 (1997) arXiv:astro-ph/9610219.

[13] K. Enqvist and M. S. Sloth, Nucl. Phys. B 626, 395 (2002) arXiv:hep-ph/0109214.

[14] D. H. Lyth and D. Wands, Phys. Lett. B 524, 5 (2002) arXiv:hep-ph/0110002 .

[15] T. Moroi and T. Takahashi, Phys. Lett. B 522, 215 (2001) [Erratum-ibid. B 539, 303 (2002)] arXiv:hep-ph/0110096; Phys. Rev. D 66, 063501 (2002) arXiv:hep-ph/0206026.

[16] K. Dimopoulos and D. H. Lyth, arXiv:hep-ph/0209180 K. Enqvist and A. Mazumdar, Phys. Rept. 380, 99 (2003) arXiv:hep-ph/0209244.

[17] N. Bartolo and A. R. Liddle, Phys. Rev. D 65, 121301 (2002) arXiv:astro-ph/0203076. D. Wands, N. Bartolo, S. Matarrese and A. Riotto, Phys. Rev. D 66, 043520 (2002) arXiv:astro-ph/0205253. M. Postma, Phys. Rev. D 67, 063518 (2003) arXiv:hep-ph/0212005. M. Giovannini, Phys. Rev. D 67, 123512 (2003) arXiv:hep-ph/0301264.

[18] D. Wands, K. A. Malik, D. H. Lyth and A. R. Liddle, Phys. Rev. D 62, 043527 (2000) arXiv:astro-ph/0003278.

[19] D. H. Lyth and D. Wands, arXiv:astro-ph/0306498

[20] D. H. Lyth, C. Ungarelli and D. Wands, Phys. Rev. D 67, 023503 (2003) arXiv:astro-ph/0208055.

[21] D. H. Lyth and D. Wands, arXiv:astro-ph/0306500

[22] K. A. Malik, D. Wands and C. Ungarelli, Phys. Rev. D 67, 063516 (2003) arXiv:astro-ph/0211602.

[23] H. Kodama and M. Sasaki, Prog. Theor. Phys. Suppl. 78 (1984) 1.

[24] J. M. Bardeen, P. J. Steinhardt and M. S. Turner, Phys. Rev. D 28, 679 (1983).

[25] J. M. Bardeen, DOE/ER/40423-01-C8 Lectures given at 2nd Guo Shou-jing Summer School on Particle Physics and Cosmology, Nanjing, China, Jul 1988.

[26] Y. Nambu and A. Taruya, Prog. Theor. Phys. 97, 83 (1997) arXiv:gr-qc/9609029; F. Finelli and R. H. Brandenberger, Phys. Rev. Lett. 82, 1362 (1999) arXiv:hep-ph/9809490; A. R. Liddle, D. H. Lyth, K. A. Malik and D. Wands, Phys. Rev. D 61, 103509 (2000) arXiv:hep-ph/9912473.

[27] M. Bucher, K. Moodley and N. Turok, Phys. Rev. D 62, 083508 (2000) arXiv:astro-ph/9904231.

[28] D. Wands, N. Bartolo, S. Matarrese and A. Riotto, Phys. Rev. D 66, 043520 (2002) arXiv:astro-ph/0205253.

[29] E. Komatsu and D. N. Spergel, Phys. Rev. D 63, 063002 (2001) arXiv:astro-ph/0005036.

[30] N. Bartolo, S. Matarrese and A. Riotto, arXiv:astro-ph/0309692

[31] J. M. Bardeen, Phys. Rev. D 22 (1980) 1882.

[32] J. Garcia-Bellido and D. Wands, Phys. Rev. D 53, 5437 (1996) arXiv:astro-ph/9511029.

\section{APPENDIX A: GAUGE-INVARIANT ADIABATIC AND ENTROPY PERTURBATIONS}

In a multi-component system it is often useful to re-write the coupled perturbation equations in terms of the instantaneous overall curvature perturbation (often called the adiabatic perturbation) and the relative entropy perturbations between fluids. In this appendix we follow the treatment of Ref. [22] and the reader is referred to this source for more details. Throughout this paper we work in the large scale limit, i.e., neglecting gradient terms. 


\section{Evolution of curvature and entropy perturbations}

The gauge-invariant definition of the total curvature perturbation on uniform-density hypersurfaces is [18, 24, 31]

$$
\zeta \equiv-\psi-H \frac{\delta \rho}{\dot{\rho}}
$$

where $\psi$ and $\delta \rho$ are the gauge-dependent curvature and density perturbations. The evolution of the curvature perturbation is given on large scales by [18, 22, 32]

$$
\dot{\zeta}=-\frac{H}{\rho+P} \delta P_{\text {nad }}
$$

where the non-adiabatic pressure perturbation is $\delta P_{\text {nad }} \equiv \delta P-c_{\mathrm{s}}^{2} \delta \rho$ and the adiabatic sound speed is $c_{\mathrm{s}}^{2}=\dot{P} / \dot{\rho}$. Thus the total curvature perturbation is constant on large scales for purely adiabatic perturbations.

In the presence of more than one fluid, the total non-adiabatic pressure perturbation, $\delta P_{\text {nad }}$, may be split into two parts,

$$
\delta P_{\text {nad }} \equiv \delta P_{\text {intr }}+\delta P_{\text {rel }}
$$

The first part is due to the intrinsic entropy perturbation of each fluid

$$
\delta P_{\text {intr }}=\sum_{\alpha} \delta P_{\text {intr }, \alpha}
$$

where the intrinsic non-adiabatic pressure perturbation of the $\alpha$-fluid is given by

$$
\delta P_{\text {intr }, \alpha} \equiv \delta P_{\alpha}-c_{\alpha}^{2} \delta \rho_{\alpha}
$$

where $c_{\alpha}^{2} \equiv \dot{P}_{\alpha} / \dot{\rho}_{\alpha}$ is the adiabatic sound speed of that fluid. The second part of the total non-adiabatic pressure perturbation (A3) is due to the relative entropy perturbation between different fluids,

$$
\delta P_{\text {rel }} \equiv-\frac{1}{6 H \dot{\rho}} \sum_{\alpha, \beta} \dot{\rho}_{\alpha} \dot{\rho}_{\beta}\left(c_{\alpha}^{2}-c_{\beta}^{2}\right) \mathcal{S}_{\alpha \beta}
$$

where $\mathcal{S}_{\alpha \beta}$ is the relative entropy (or isocurvature) perturbation, Eq. (28),

$$
\mathcal{S}_{\alpha \beta}=-3 H\left(\frac{\delta \rho_{\alpha}}{\dot{\rho}_{\alpha}}-\frac{\delta \rho_{\beta}}{\dot{\rho}_{\beta}}\right) .
$$

Note, that Eq. (A6) corrects a sign error in Eq. (2.31) of Ref. [22].

The evolution equation for the relative entropy perturbation $\mathcal{S}_{\alpha \beta}$ on large scales is given by [22]

$$
\dot{\mathcal{S}}_{\alpha \beta}=3 H\left(\frac{3 H \delta P_{\mathrm{intr}, \alpha}-\delta Q_{\mathrm{intr}, \alpha}-\delta Q_{\mathrm{rel}, \alpha}}{\dot{\rho}_{\alpha}}-\frac{3 H \delta P_{\mathrm{intr}, \beta}-\delta Q_{\mathrm{intr}, \beta}-\delta Q_{\mathrm{rel}, \beta}}{\dot{\rho}_{\beta}}\right),
$$

where the intrinsic non-adiabatic energy transfer perturbation is defined as [22]

$$
\delta Q_{\text {intr }, \alpha} \equiv \delta Q_{\alpha}-\frac{\dot{Q}_{\alpha}}{\dot{\rho}_{\alpha}} \delta \rho_{\alpha}
$$

and the relative non-adiabatic energy transfer is defined as

$$
\delta Q_{\mathrm{rel}, \alpha}=\frac{Q_{\alpha} \dot{\rho}}{2 \rho}\left(\frac{\delta \rho_{\alpha}}{\dot{\rho}_{\alpha}}-\frac{\delta \rho}{\dot{\rho}}\right)=-\frac{Q_{\alpha}}{6 H \rho} \sum_{\beta} \dot{\rho}_{\beta} \mathcal{S}_{\alpha \beta}
$$

For fluids, such as radiation or non-relativistic matter in our curvaton model, with definite equation of state, $P_{\alpha}\left(\rho_{\alpha}\right)$, the intrinsic non-adiabatic pressure perturbation in Eq. (A4) is zero. Hence the total non-adiabatic pressure perturbation (A6) for our model can be written as

$$
\delta P_{\text {nad }}=\delta P_{\text {rel }}=\frac{\dot{\rho}_{\gamma}}{9 H \dot{\rho}}\left(\dot{\rho}_{\mathrm{m}} \mathcal{S}_{\mathrm{m} \gamma}+\dot{\rho}_{\sigma} \mathcal{S}_{\sigma \gamma}\right) .
$$


Hence the evolution equation (A2) for $\zeta$ is

$$
\dot{\zeta}=\frac{H \dot{\rho}_{\gamma}}{3 \dot{\rho}^{2}}\left(\dot{\rho}_{\sigma} \mathcal{S}_{\sigma \gamma}+\dot{\rho}_{\mathrm{m}} \mathcal{S}_{\mathrm{m} \gamma}\right)
$$

The intrinsic energy transfer perturbation, following from Eq. (21), are

$$
\begin{aligned}
\delta Q_{\mathrm{intr}, \sigma} & =0 \\
\delta Q_{\mathrm{intr}, \gamma} & =-\frac{\Gamma_{1}}{3 H} \dot{\rho}_{\sigma} \mathcal{S}_{\sigma \gamma}, \\
\delta Q_{\mathrm{intr}, \mathrm{m}} & =-\frac{\Gamma_{2}}{3 H} \dot{\rho}_{\sigma} \mathcal{S}_{\sigma \mathrm{m}},
\end{aligned}
$$

while the relative energy transfer perturbations are

$$
\begin{aligned}
\delta Q_{\mathrm{rel}, \sigma} & =\frac{\left(\Gamma_{1}+\Gamma_{2}\right) \rho_{\sigma}}{6 H \rho}\left(\dot{\rho}_{\gamma} \mathcal{S}_{\sigma \gamma}+\dot{\rho}_{\mathrm{m}} \mathcal{S}_{\sigma \mathrm{m}}\right), \\
\delta Q_{\mathrm{rel}, \gamma} & =\frac{\Gamma_{1} \rho_{\sigma}}{6 H \rho}\left(\dot{\rho}_{\sigma} \mathcal{S}_{\sigma \gamma}+\dot{\rho}_{\mathrm{m}} \mathcal{S}_{\mathrm{m} \gamma}\right), \\
\delta Q_{\mathrm{rel}, \mathrm{m}} & =\frac{\Gamma_{2} \rho_{\sigma}}{6 H \rho}\left(\dot{\rho}_{\sigma} \mathcal{S}_{\sigma \mathrm{m}}-\dot{\rho}_{\gamma} \mathcal{S}_{\mathrm{m} \gamma}\right) .
\end{aligned}
$$

Hence the evolution of the relative entropy perturbations can be written as

$$
\begin{aligned}
& \dot{\mathcal{S}}_{\sigma \gamma}=\frac{1}{2 \rho}\left[\Gamma_{1} \rho_{\sigma} \frac{\dot{\rho}_{\mathrm{m}}}{\dot{\rho}_{\gamma}} \mathcal{S}_{\mathrm{m} \gamma}-\left(\Gamma_{1}+\Gamma_{2}\right) \rho_{\sigma} \frac{\dot{\rho}_{\mathrm{m}}}{\dot{\rho}_{\sigma}} \mathcal{S}_{\sigma \mathrm{m}}-\left(2 \Gamma_{1} \rho \frac{\dot{\rho}_{\sigma}}{\dot{\rho}_{\gamma}}-\Gamma_{2} \rho_{\sigma} \frac{\dot{\rho}_{\gamma}}{\dot{\rho}_{\sigma}}\right) \mathcal{S}_{\sigma \gamma}\right], \\
& \dot{\mathcal{S}}_{\mathrm{m} \gamma}=\left(\Gamma_{1} \frac{\dot{\rho}_{\mathrm{m}}}{\dot{\rho}_{\gamma}}-\Gamma_{2} \frac{\dot{\rho}_{\gamma}}{\dot{\rho}_{\mathrm{m}}}\right) \frac{\rho_{\sigma}}{2 \rho} \mathcal{S}_{\mathrm{m} \gamma}+\Gamma_{2}\left(1-\frac{\rho_{\sigma}}{2 \rho}\right) \frac{\dot{\rho}_{\sigma}}{\dot{\rho}_{\mathrm{m}}} \mathcal{S}_{\sigma \mathrm{m}}-\Gamma_{1}\left(1-\frac{\rho_{\sigma}}{2 \rho}\right) \frac{\dot{\rho}_{\sigma}}{\dot{\rho}_{\gamma}} \mathcal{S}_{\sigma \gamma}, \\
& \dot{\mathcal{S}}_{\sigma \mathrm{m}}=-\left(\Gamma_{1}+\Gamma_{2}\right) \frac{\rho_{\sigma}}{2 \rho} \frac{\dot{\rho}_{\gamma}}{\dot{\rho}_{\sigma}} \mathcal{S}_{\sigma \gamma}-\left[\Gamma_{2}\left(1-\frac{\rho_{\sigma}}{2 \rho}\right) \frac{\dot{\rho}_{\sigma}}{\dot{\rho}_{\mathrm{m}}}+\left(\Gamma_{1}+\Gamma_{2}\right) \frac{\rho_{\sigma}}{2 \rho} \frac{\dot{\rho}_{\mathrm{m}}}{\dot{\rho}_{\sigma}}\right] \mathcal{S}_{\sigma \mathrm{m}}-\Gamma_{2} \frac{\rho_{\sigma}}{2 \rho} \frac{\dot{\rho}_{\gamma}}{\dot{\rho}_{\mathrm{m}}} \mathcal{S}_{\mathrm{m} \gamma} .
\end{aligned}
$$

Note that we can always identify a trivial solution to Eqs. A19 A21) with all the entropy perturbations $\mathcal{S}_{\alpha \beta}=0$ which corresponds to the adiabatic mode and leaves $\zeta=$ constant in Eq. A12), even in a system such as this including energy transfer between different components.

\section{Singular and Non-Singular Hypersurfaces}

From Eq. A7 we see that the relative entropy perturbation $\mathcal{S}_{\alpha \beta}$ becomes singular whenever $\dot{\rho}_{\alpha}=0$. This can occur if we have multiple fluids with energy transfer between them whenever $Q_{\alpha}=3 H\left(\rho_{\alpha}+P_{\alpha}\right)$. This is because the uniform $\alpha$-fluid hypersurfaces become ill-defined to first-order whenever $\rho_{\alpha}$ is stationary, i.e., the first-derivative vanishes.

Thus a divergence in a gauge-invariant quantity such as $\mathcal{S}_{\alpha \beta}$ need not signal the breakdown of perturbation theory, but instead may indicate that a particular choice of hypersurface was not well-defined [26]. This is indeed the case in the curvaton model studied in this paper: we see that the density perturbations on uniform-curvature hypersurfaces remain finite even when $\dot{\rho}_{\alpha}=0$.

From the definition of $\mathcal{S}_{\alpha \beta}$, Eq. [A7) above, we see that we can construct a non-singular quantity

$$
\Delta_{\alpha \beta} \equiv \dot{\rho}_{\alpha} \dot{\rho}_{\beta} \mathcal{S}_{\alpha \beta}=-3 H\left(\dot{\rho}_{\beta} \delta \rho_{\alpha}-\dot{\rho}_{\alpha} \delta \rho_{\beta}\right) .
$$

Since the definition of $\mathcal{S}_{\alpha \beta}$ is gauge invariant then so is $\Delta_{\alpha \beta}$.

The evolution equation for the total curvature perturbation on large scales, Eq. (A2) can then be written in terms of $\Delta_{\alpha \beta}$ as

$$
\dot{\zeta}=\frac{1}{3 H(\rho+P)^{2}} \sum_{\alpha}\left\{H \delta P_{\mathrm{intr}, \alpha}-c_{\alpha}^{2} \sum_{\beta} \Delta_{\alpha \beta}\right\} .
$$

The uniform total density hypersurfaces, on which $\zeta$ is defined, always remain well defined, since $\dot{\rho} \neq 0$ after inflation. 